\title{
Nomogram for short-term outcome assessment in AChR subtype generalized myasthenia gravis
}

\author{
Rui Zhao ${ }^{1 \dagger}$, Ying Wang ${ }^{2 \dagger}$, Xiao Huan ${ }^{1}$, Huahua Zhong ${ }^{1}$, Zhirui Zhou ${ }^{3}$, Jianying Xi ${ }^{1}$, Yuwei Da ${ }^{4}$, Lin Lei ${ }^{4}$, \\ Ting Chang ${ }^{5}$, Zhe Ruan ${ }^{5}$, Lijun Luo ${ }^{6}$, Shengnan Li ${ }^{6}$, Huan Yang ${ }^{7}, \mathrm{Yi} \mathrm{Li}^{7}$, Sushan Luo ${ }^{*^{*}}$ (D) and Chongbo Zhao ${ }^{1^{*}}$
}

\begin{abstract}
Background: An accurate prediction for prognosis can help in guiding the therapeutic options and optimizing the trial design for generalized myasthenia gravis ( $g M G)$. We aimed to develop and validate a predictive nomogram to assess the short-term outcome in patients with the anti-acetylcholine receptor (AChR) subtype gMG.

Methods: We retrospectively reviewed 165 patients with AChR subtype gMG who were immunotherapy naïve at the first visit from five tertiary centers in China. The short-term clinical outcome is defined as the achievement of minimal symptom expression (MSE) at 12 months. Of them, $120 \mathrm{gMG}$ patients from Huashan Hospital were enrolled to form a derivation cohort $(n=96)$ and a temporal validation cohort $(n=24)$ for the nomogram. Then, this nomogram was externally validated using 45 immunotherapy naïve AChR subtype gMG from the other four hospitals. Multivariate logistic regression was used to screen independent factors and construct the nomogram.

Results: MSE was achieved in 70 (72.9\%), 20 (83.3\%), and 33 (73.3\%) patients in the training, temporal validation, and external validation cohort, respectively. The duration $\leq 12$ months $(p=0.021)$, ocular score $\leq 2(p=0.006)$, QMG score $>13(p=0.008)$, and gross motor score $\leq 9(p=0.006)$ were statistically associated with MSE in AChR subtype gMG. The nomogram has good performance in predicting MSE as the concordance indexes are $0.81(95 \% \mathrm{Cl}$, $0.72-0.90)$ in the development cohort, $0.944(95 \% \mathrm{Cl}, 0.83-1.00)$ in the temporal validation cohort, and $0.773(95 \% \mathrm{Cl}$, $0.63-0.92)$ in the external validation cohort.
\end{abstract}

Conclusion: The nomogram achieved an optimal prediction of MSE in AChR subtype gMG patients using the baseline clinical characters.

Keywords: Generalized myasthenia gravis, Anti-acetylcholine receptor antibody, Minimal symptom expression, Nomogram

\section{Background}

Myasthenia gravis (MG) is an autoimmune disorder characterized by pathological autoantibody-mediated transmission defect in neuromuscular junctions (NMJ) of

\footnotetext{
*Correspondence: luosushan@fudan.edu.cn; zhao_chongbo@fudan.edu.cn

${ }^{\dagger}$ Rui Zhao and Ying Wang contributed equally to this work

${ }^{1}$ Department of Neurology, Huashan Hospital Fudan University, No.12 Middle Wulumuqi Road, Shanghai 200040, China

Full list of author information is available at the end of the article
}

ocular, bulbar, limb, respiratory, and axial muscles. It can be further divided into different subgroups according to the presentations, antibody specificity, and onset age due to the clinical heterogeneity [1]. Based on the involved muscle, there are approximately $80 \%$ of patients develop generalized weakness [2]. Of these generalized myasthenia gravis (gMG) patients, $85 \%$ are seropositive for antiacetylcholine receptor (AChR) antibodies [3]. Therefore, anti-AChR antibody-positive gMG patients account for

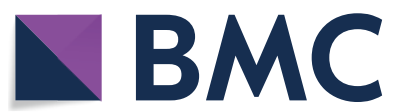

(c) The Author(s) 2021. This article is licensed under a Creative Commons Attribution 4.0 International License, which permits use, sharing, adaptation, distribution and reproduction in any medium or format, as long as you give appropriate credit to the original author(s) and the source, provide a link to the Creative Commons licence, and indicate if changes were made. The images or other third party material in this article are included in the article's Creative Commons licence, unless indicated otherwise in a credit line to the material. If material is not included in the article's Creative Commons licence and your intended use is not permitted by statutory regulation or exceeds the permitted use, you will need to obtain permission directly from the copyright holder. To view a copy of this licence, visit http://creativeco mmons.org/licenses/by/4.0/. The Creative Commons Public Domain Dedication waiver (http://creativecommons.org/publicdomain/ zero/1.0/) applies to the data made available in this article, unless otherwise stated in a credit line to the data. 
the majority of MG and also are the main participants in the clinical trials for new immunotherapies.

Therapeutic response and the outcome for gMG patients are critical concerns in clinical practice. Conventional immunotherapies for gMG include corticosteroids and immunomodulatory agents (e.g., azathioprine, mycophenolate mofetil, methotrexate, cyclosporine, and tacrolimus) [4]. A predictive model has been developed for evaluating the corticosteroid-induced initial worsening in a prospective cohort [5]. However, there is still an unmet need for developing a model to predict the clinical outcome for gMG patients [6], especially in the era with emerging therapies development such as eculizumab and neonatal Fc receptor inhibitors [7-9]. For the MG patients who are likely to achieve remission, the benefit from the excessive treatment maybe not be cost-effective [10]. Longitudinal studies provided evidence that approximately $75 \%$ of MG patients had an optimal outcome with remission, confined ocular involvement, or mild weakness, while only $7 \%$ achieved complete stable remission within a decade [1]. Recently, minimal symptom expression (MSE) that is defined as the patient-reported MG activity of daily living (MG-ADL) scale $0-1$ has been used to evaluate the clinical efficacy of efgartigimod in gMG [11]. In comparison to other measures, MSE may provide a more representative outcome measurement for the majority of gMG patients.

In this study, we aim to develop and validate a nomogram for predicting the clinical short-term outcome for gMG patients using the baseline clinical characteristics.

\section{Methods}

\section{Study design and patient recruitment}

There are 1193 MG patients registered in a tertiary referral diagnostic center in Huashan Hospital from August 8, 2012, through December 18, 2020. The inclusion criteria were (1) onset symptoms and signs compatible with gMG; (2) immunotherapy naive at baseline; (3) seropositive for anti-AChR antibody; (4) MG-ADL score $>1$ at baseline; (5) follow-up period longer than half a year from baseline; (6) exclusion of other MG mimicking diseases including Lambert-Eaton myasthenic syndrome, peripheral neuropathy, myopathies, and motor neuron diseases. Eligible patients with the integrated baseline data recruited from February 13, 2017, through August 2, 2019, were included in the training cohort for the development of the nomogram, and those recruited from August 2, 2019, through March 13, 2020, were included into the temporal validation cohort. Then, the nomogram was externally validated using 45 anti-AChR antibodypositive gMG patients who have not received immunotherapy from May 2015 to May 2021 at 4 tertiary centers in China (Xiangya Hospital, Xuanwu Hospital, Tangdu Hospital, and Wuhan No.1 Hospital).

The clinical baseline variables include gender, age at onset, the comorbidities of autoimmune disease, and disease duration. The age at onset of MG is classified into three subgroups including early-onset (10-49 years), lateonset (50-64 years), and elderly-onset (65 years or older) [12]. The concurrent autoimmune diseases identified in our cohort include Graves' disease, Hashimoto's autoimmune thyroiditis, type 1 diabetes mellitus, immune thrombocytopenic purpura, autoimmune hemolytic anemia, and vitiligo [13]. The disease duration is defined as the period from the onset of weakness symptoms of MG to the first visit to our hospital. The MG associated clinical features include Myasthenia Gravis Foundation of America (MGFA) classification, thymoma concurrence, history of thymectomy, MG worsening, anti-AChR antibodies titers, pyridostigmine dosage, manual muscle test (MMT) score, MG-ADL score, and the related subscores (bulbar, respiratory, ocular, and limb score), and quantitative myasthenia gravis (QMG) score and the related subscores (extraocular muscle, bulbar muscle, gross motor, and axial motor score). The presence of thymoma is determined by a computed tomography scan. MG worsening is defined as a substantial exacerbation in muscle weakness and fatigability, or increased medication [14]. The anti-AChR antibodies titer was measured by enzyme-linked immunosorbent assay (ELISA, Euroimmun, Lübeck, Germany) and the cut-off value was $0.50 \mathrm{nmol} / \mathrm{L}$.

We divided the total MG-ADL score into four subscores: (1) Ocular score: double vision and eyelid droop; (2) Bulbar score: talking, chewing, and swallowing activities; (3) Respiratory score: the activity of breathing; (4) Limb score: the ability to brush teeth or comb hair, and arise from a chair. For QMG score, it was divided into 5 subscores: (1) Extraocular muscle score: first three items (double vision on lateral gaze, ptosis, and facial muscles); (2) Bulbar muscle: score fourth and fifth items (swallowing $4 \mathrm{oz}$. water, and the onset of dysarthria); (3) Gross motor score: sixth, seventh, ninth, and tenth, twelfth, and thirteenth items (arms outstretched, hands grip, and legs outstretched); (4) Axial motor score: eleventh item (head lifted); (5) Respiratory score: eighth item (Vital capacity, $\%$ predicted).

\section{Statistical analysis}

Our analysis showed that the continuous demographic characteristics data in this study were not normally distributed. The missing data of thymoma, thymectomy, anti-AChR Abs titer, and pyridostigmine dosage account for less than $10 \%$. These missing data were missed at random and replaced by the average of the observed values. 
Continuous variables were expressed as medians (quartiles) and compared between groups using the MannWhitney $U$ test. Categorical variables were expressed as frequencies (percentages) and were tested using the $\chi^{2}$ test or Fisher exact test. To determine the cut-off values of the continuous variable, we created receiver operating characteristic curves for "MSE" and defined them as the points on the ROC curve where Youden's index reached the highest. The significance of each variable in the training cohort was analyzed using univariate and multivariate logistic regression analyses. Variables showing statistical $(P<0.1)$ and clinical significance of the univariate analysis were included in the multivariate logistic regression analysis to develop the nomogram to predict whether a patient would achieve MSE.

The variance inflation factors (VIFs) were generated to examine individual predictors for potentially strong contributions to multicollinearity. The discrimination performance of this nomogram was measured by the concordance index (C-index) in the training and validation cohorts. The Hosmer-Lemeshow test was applied to assess the agreement between nomogram predicted and observed probabilities. All analyses were performed using IBM SPSS version 20.0 (SPSS Company, Chicago, IL, USA) and R software ( $\mathrm{R}$ version 4.0.3, USA).

\section{Results}

\section{Patient demographic characteristics}

A total of 1193 MG patients have been initially registered in our referral center-based database. According to the inclusion flowchart, we finally enrolled $120 \mathrm{AChR}$ subtype gMG patients with no immunotherapies at the baseline registry (Fig. 1). Of these gMG patients, 96 and 24 patients were then included in the training and temporal validation cohort splitting by time. Besides, $45 \mathrm{AChR}$ subtype gMG patients from the other 4 centers were enrolled for external validation.

The baseline clinical characteristics and the outcome of MG patients in the training and temporal validation cohort were comparable, except for the differences in the respiratory score $(p=0.035)$, and respiratory muscle score $(p=0.002)$ (Table 1$)$. In the development set, 70 patients (72.9\%) achieved MSE and the median disease duration was $7(3.0-30.5)$ months. At baseline, 34\% of patients were diagnosed to have concurrent thymoma and $24 \%$ had undergone thymectomy in the development set. The ADL and QMG scores of the development cohort were 5 (4.0-8.0) and 11 (9.0-14.0), respectively.

The clinical characteristics of the external validation and development groups are summarized in Table 2 . The frequency of MSE was similar for the development $(72.9 \%)$ and external validation groups (73.3\%), whereas there were some differences between these groups regarding the frequency of thymectomy, disease duration, anti-AChR Abs titer, MMT score, bulbar score, bulbar muscle score, and gross motor score.

\section{Short-term clinical outcome assessment}

MSE status was achieved in 70 (72.9\%), 20 (83.3\%), and 33 (73.3\%) patients in the training, temporal validation, and external validation cohorts at 12 months after baseline recruitment. For the patients who did not achieve MSE, the median ADL scores were 3 (range 3-6), 4.5 (range $3.25-5$ ), and 2.5 (range 2-7) in the training, temporal validation, and external validation cohorts, respectively.

In the training and temporal validation groups in Huashan Hospital, the initial dose and dose-escalating manner of prednisone depended on the physician's decision. The final oral prednisone dose for each patient was at $0.8 \mathrm{mg}$ to $1 \mathrm{mg} / \mathrm{kg}$ and azathioprine, tacrolimus, or mycophenolate mofetil as immunosuppressants concurrent with oral prednisone. Three patients had received rescue therapies including immunoglobulin and plasma exchange.

\section{Nomogram development and validation}

We identified three risk factors significantly associated with MSE including duration, ocular score, and gross motor score $(p<0.1)$ (Table 3). Considering the clinical significance, we also included the QMG score $(p=0.155)$ along with these statistically significant variables into the multivariate logistic regression. All these above variables were independently associated with MSE $(p<0.05)$, with results reported as odds ratio $(95 \% \mathrm{CI})$, duration $\leq 12$ months (3.45 [1.23-10.24]), ocular score $\leq 2$ (6.00 [1.82 to 24.58]), QMG score $>13$ (11.95 [2.31 to 95.82]), and gross motor score $\leq 9$ (10.82 [2.22-69.13]). The VIFs of them were $1.01,1.25,1.78$, and 1.84 respectively, suggesting that there was no multiple collinearity among the four independent risk factors. We then used these four factors to establish an individualized prediction nomogram, which can calculate the total point for each gMG patient with anti-AChR antibodies and converted it to predicted probabilities of MSE (Fig. 2). This nomogram was then validated in both the temporal validation cohort derived from Huashan Hospital and the external validation cohort.

The ability of this nomogram to differentiate between patients who do or do not achieve MSE is excellent as the C-indexes are 0.810 (95\% CI, 0.72-0.90), 0.944 (95\% CI $0.83-1.00$ ), and 0.773 (95\% CI, 0.63-0.92) in the development, temporal validation, and external validation cohorts, respectively (Fig. 3). Besides, the p-values of the Hosmer-Lemeshow test are 0.98, 0.99, and 0.61 for the development, temporal validation, and external 


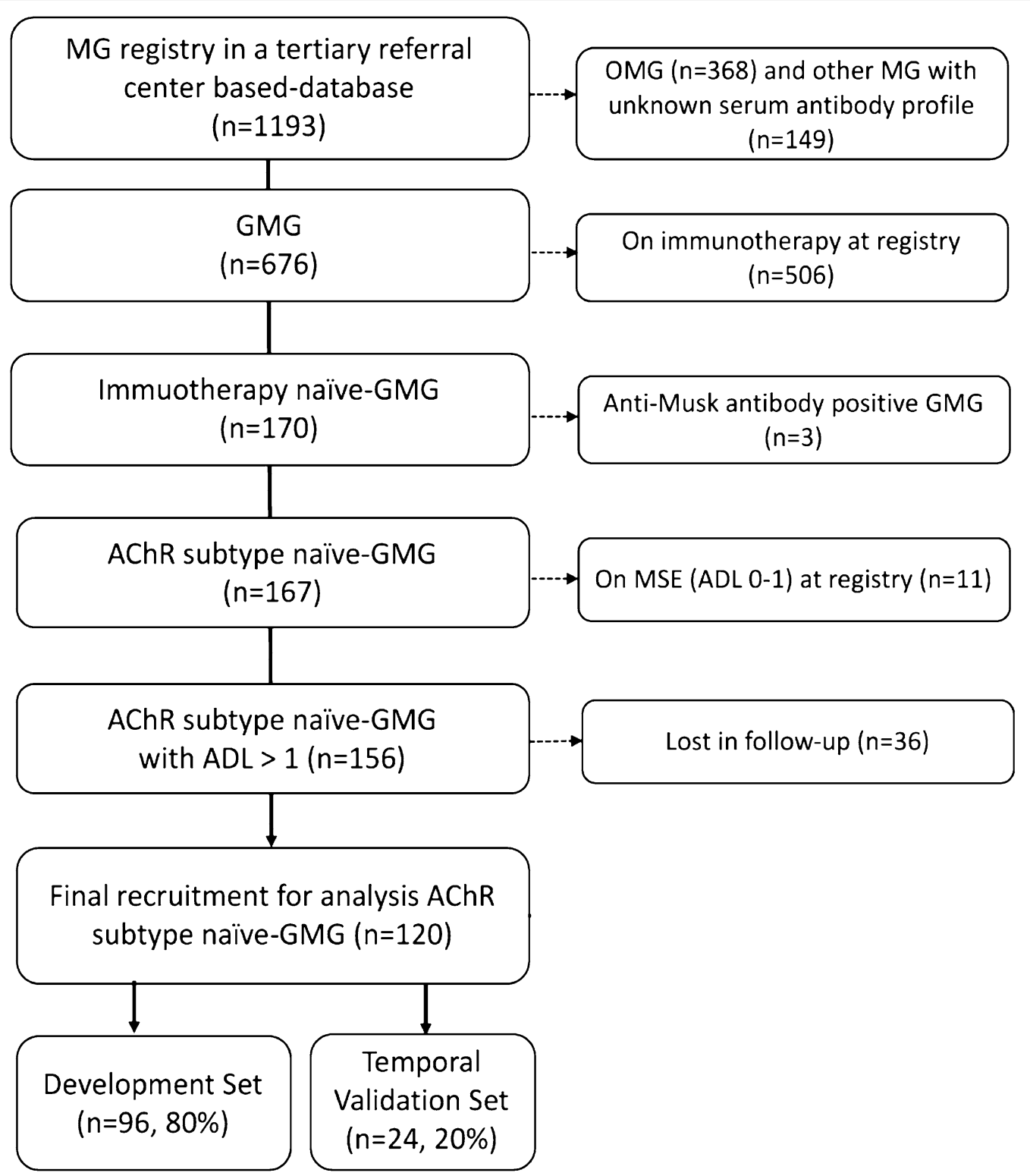

Fig. 1 Flow diagram of the enrollment of study participants and the classification of training and temporal validation set

validation sets, which indicates good agreement between nomogram predicted and observed probabilities.

\section{Discussion}

To optimize the clinical management, the need to identify the patients with good prognoses using the baseline characteristics is unmet. In this multicenter study, we suggest that disease duration $\leq 12$ months, ocular score $\leq 2$, QMG score $>13$, and gross motor score $\leq 9$ before immunosuppressants administration are significant predictors for reaching the status of MSE in AChR subtype gMG patients.
For patients with anti-AChR antibodies, these antibodies bind and activate the complement cascade at the NMJ, resulting in the postsynaptic folds degeneration of skeletal muscle [12]. It has been shown that chronic anticholinesterase treatment in rats could destroy the postsynaptic membrane [15]. Around 80 patients (83.3\%) included in the training cohort have only administered pyridostigmine before the first visit. From our study, we indicated that the patients who had a duration from onset to immunosuppressive therapies shorter than 12 months had a better outcome, which may be due to an alleviate NMJ destruction. A systematic review also identified the 
Table 1 The Baseline Demographic Characteristics and Outcome of the Development and Validation Set

\begin{tabular}{|c|c|c|c|c|}
\hline Variables & Subgroups & $\begin{array}{l}\text { Development set }(n=96) \text { No. of } \\
\text { patients (\%) Median (range) }\end{array}$ & $\begin{array}{l}\text { Temporal validation set }(n=24) \text { No. } \\
\text { of patients (\%) Median (range) }\end{array}$ & $P$ value \\
\hline \multirow[t]{2}{*}{ Outcome } & Non MSE & $26(27.1)$ & $4(16.7)$ & 0.292 \\
\hline & MSE & $70(72.9)$ & $20(83.3)$ & \\
\hline \multirow[t]{2}{*}{ Gender } & Male & $46(47.9)$ & $15(62.5)$ & 0.201 \\
\hline & Female & $50(52.1)$ & $9(37.5)$ & \\
\hline \multirow[t]{3}{*}{ Age at onset } & EOMG & $62(64.6)$ & $16(66.7)$ & 0.815 \\
\hline & LOMG & $25(26.0)$ & $5(20.8)$ & \\
\hline & Elderly-onset MG & $9(9.6)$ & $3(12.5)$ & \\
\hline \multirow[t]{3}{*}{ MGFA classification } & $\|$ & $62(64.6)$ & $16(66.7)$ & 0.999 \\
\hline & III & $29(30.2)$ & $7(29.2)$ & \\
\hline & IV & $5(5.2)$ & $1(4.2)$ & \\
\hline \multirow[t]{2}{*}{ Thymoma } & No & $62(66.0)$ & $19(79.2)$ & 0.213 \\
\hline & Yes & $32(34.0)$ & $5(20.8)$ & 0.633 \\
\hline \multirow[t]{2}{*}{ Thymectomy } & No & $70(74.5)$ & $19(79.2)$ & \\
\hline & Yes & $24(25.5)$ & $5(20.8)$ & \\
\hline \multirow[t]{2}{*}{ Worsening } & No & $24(25.0)$ & $9(37.5)$ & 0.22 \\
\hline & Yes & $72(75.0)$ & $15(62.5)$ & \\
\hline \multirow[t]{2}{*}{ Autoimmune disease } & No & $86(89.6)$ & $20(83.3)$ & 0.619 \\
\hline & Yes & $10(10.4)$ & $4(16.7)$ & \\
\hline Disease duration, months & & $7(3.0-30.5)$ & $4(2.0-14.5)$ & 0.305 \\
\hline Anti-AChR Abs titer, nmol/L & & $6(2.6-10.3)$ & $8(2.6-12.4)$ & 0.316 \\
\hline Pyridostigmine dosage, mg/day & & $180(90.0-180.0)$ & $180(180.0-180.0)$ & 0.557 \\
\hline MMT score & & $14(7.0-19.0)$ & $14(7.3-19.8)$ & 0.743 \\
\hline MG-ADL score & & $5(4.0-8.0)$ & $5(4.0-7.0)$ & 0.377 \\
\hline Bulbar score & & $2(1.0-3.0)$ & $1(0-2.8)$ & 0.145 \\
\hline Respiratory score & & $0(0-1.0)$ & $0(0-0)$ & $0.035^{*}$ \\
\hline Limb score & & $1(0-2.0)$ & $0(0-1.0)$ & 0.103 \\
\hline Ocular score & & $3(1.0-4.0)$ & $3(2.0-4.0)$ & 0.182 \\
\hline QMG score & & $11(9.0-14.0)$ & $12(7.0-16.0)$ & 0.638 \\
\hline Extraocular muscle score & & $3(1.0-4.0)$ & $4(2.0-5.0)$ & 0.038 \\
\hline Bulbar muscle score & & $0(0-1.0)$ & $1(0-2.0)$ & 0.110 \\
\hline Respiratory muscle score & & $0(0-1.0)$ & $0(0-0)$ & $0.002^{*}$ \\
\hline Gross motor score & & $6(4.0-8.0)$ & $6(2.5-8.0)$ & 0.220 \\
\hline Axial motor score & & $1(1.0-2.0)$ & $1(1.0-2.0)$ & 0.869 \\
\hline
\end{tabular}

MSE minimal symptom expression, EOMG early-onset myasthenia gravis, LOMG late-onset myasthenia gravis, MG myasthenia gravis, MGFA Myasthenia Gravis Foundation of America, $A C h R$ acetylcholine receptor, $A b s$ antibodies, $M M T$ manual muscle test, $M G-A D L$ myasthenia gravis-activity of daily living, $Q M G$ quantitative myasthenia gravis

*Statistical significance $(a=0.05)$

duration between onset and diagnosis ( $<1$ year) was a strong predictor of remission for MG patients [6].

We also found that MG patients whose baseline QMG score more than 13 were more likely to achieve MSE. Previous logistic regression analysis had also confirmed that the high baseline QMG score serves as an independent predictor of response to immunotherapy [16]. As a result, these patients with more severe weakness symptoms measured by the QMG appear to respond better to treatments and have good prognoses.
QMG is a valid subject scale to assess the severity of the weakness. However, the items are linearly scored and not weighted. Based on the clinical practicability, though the signs of poor vital capacity and spontaneous ptosis gain the same QMG score, the degree of disability experienced by the former is more severe. As a result, MGFA also recommended "weighting" specific sub-scores of the QMG [17]. In this study, QMG has been divided into five functional subscores and the gross motor score $\leq 9$ was a significant risk factor for MSE in MG. In an observational 
Table 2 The Baseline Demographic Characteristics and Outcome of the Development and External Validation Set

\begin{tabular}{|c|c|c|c|c|}
\hline Variables & Subgroups & $\begin{array}{l}\text { Development set }(n=96) \text { No. of } \\
\text { patients (\%) Median (range) }\end{array}$ & $\begin{array}{l}\text { External validation set }(n=45) \text { No. } \\
\text { of patients }(\%) \text { Median (range) }\end{array}$ & $P$ value \\
\hline \multirow[t]{2}{*}{ Outcome } & Non MSE & $26(27.1)$ & $12(26.7)$ & 0.959 \\
\hline & MSE & $70(72.9)$ & $33(73.3)$ & \\
\hline \multirow[t]{2}{*}{ Gender } & Male & $46(47.9)$ & $22(48.9)$ & 0.914 \\
\hline & Female & $50(52.1)$ & $23(51.1)$ & \\
\hline \multirow[t]{3}{*}{ Age at onset } & EOMG & $62(64.6)$ & $23(51.1)$ & 0.096 \\
\hline & LOMG & $25(26.0)$ & $12(26.7)$ & \\
\hline & Elderly-onset MG & $9(9.6)$ & $10(22.2)$ & \\
\hline \multirow[t]{3}{*}{ MGFA classification } & $\|$ & $62(64.6)$ & $21(46.7)$ & 0.128 \\
\hline & III & $29(30.2)$ & $20(44.4)$ & \\
\hline & IV & $5(5.2)$ & $4(8.9)$ & \\
\hline \multirow[t]{2}{*}{ Thymoma } & No & $62(66.0)$ & $32(71.1)$ & 0.543 \\
\hline & Yes & $32(34.0)$ & $13(28.9)$ & \\
\hline \multirow[t]{2}{*}{ Thymectomy } & No & $70(74.5)$ & $41(91.1)$ & $0.022^{*}$ \\
\hline & Yes & $24(25.5)$ & $4(8.9)$ & \\
\hline \multirow[t]{2}{*}{ Worsening } & No & $24(25.0)$ & $7(15.6)$ & 0.207 \\
\hline & Yes & $72(75.0)$ & $38(84.4)$ & \\
\hline \multirow[t]{2}{*}{ Autoimmune disease } & No & $86(89.6)$ & $37(82.2)$ & 0.222 \\
\hline & Yes & $10(10.4)$ & $8(17.8)$ & \\
\hline Disease duration, months & & $7(3.0-30.5)$ & $2(1.0-6.0)$ & $0.001^{*}$ \\
\hline Anti-AChR Abs titer, nmol/L & & $6(2.6-10.3)$ & $8(4.5-20.1)$ & $0.018^{*}$ \\
\hline Pyridostigmine dosage, mg/day & & $180(90.0-180.0)$ & $180(0-210.0)$ & 0.528 \\
\hline MMT score & & $14(7.0-19.0)$ & $50(41.5-50)^{\#}$ & $0.001^{*}$ \\
\hline MG-ADL score & & $5(4.0-8.0)$ & $6(4.0-9.5)$ & 0.151 \\
\hline Bulbar score & & $2(1.0-3.0)$ & $2(1.0-4.0)$ & $0.026^{*}$ \\
\hline Respiratory score & & $0(0-1.0)$ & $0(0-1.0)$ & 0.540 \\
\hline Limb score & & $1(0-2.0)$ & $0(0-2.0)$ & 0.651 \\
\hline Ocular score & & $3(1.0-4.0)$ & $3(2.0-4.0)$ & 0.458 \\
\hline QMG score & & $11(9.0-14.0)$ & $10(7.0-16.5)$ & 0.485 \\
\hline Extraocular muscle score & & $3(1.0-4.0)$ & $3(2.5-4.5)$ & 0.068 \\
\hline Bulbar muscle score & & $0(0-1.0)$ & $1(0-3.0)$ & $0.001^{*}$ \\
\hline Respiratory muscle score & & $0(0-1.0)$ & $0(0-1.0)$ & 0.357 \\
\hline Gross motor score & & $6(4.0-8.0)$ & $5(2.0-8.5)$ & $0.042^{*}$ \\
\hline Axial motor score & & $1(1.0-2.0)$ & $1(0-2.0)$ & 0.097 \\
\hline
\end{tabular}

MSE minimal symptom expression, EOMG early-onset myasthenia gravis, LOMG late-onset myasthenia gravis, MG myasthenia gravis, MGFA Myasthenia Gravis Foundation of America, $A C h R$ acetylcholine receptor, $A b s$ antibodies, $M M T$ manual muscle test, $M G-A D L$ myasthenia gravis-activity of daily living, $Q M G$ quantitative myasthenia gravis

* Statistical significance $(a=0.05)$

\# There are only Wuhan No.1 Hospital record the MMT score $(n=9)$

study of 2000 MG patients over sixty years, David et al. have found some MG patients who experienced remission also had mild weakness of legs or orbicularis oculi [18]. It was consistent with our study that gross motor score and ocular score were associated with MSE.

Our database documented the doses of corticosteroid or immunosuppressive drugs in gMG patients. The combination of immunosuppressive drugs was analyzed using univariate and multivariate logistic regression. However, there were $26(27 \%)$ missing values in the training cohort that were replaced by fifty multiple imputations (MIs) counterparts. The multivariable model of MIs data showed that the combination of immunosuppressive drugs $(\mathrm{p}=0.034$, OR 0.22 [0.06$0.87]$ ) with the above four risk factors was associated with MSE(Additional file 1: Table S1). Therefore, the baseline clinical characteristics other than advanced immunotherapy were vital predictors for MSE of gMG patients with anti-AChR antibodies. A recent study also revealed that the higher prednisolone dosage and the 
Table 3 Univariate and multivariate logistic regression models for minimal symptom expression in the development group

\begin{tabular}{|c|c|c|c|c|c|c|c|}
\hline \multirow[t]{2}{*}{ Variables } & \multirow[t]{2}{*}{ Subgroups } & \multicolumn{3}{|c|}{ Univariate analysis } & \multicolumn{3}{|c|}{ Multivariate analysis** } \\
\hline & & OR & $95 \% \mathrm{Cl}$ & $P$ value & $\overline{O R}$ & $95 \% \mathrm{Cl}$ & $P$ value \\
\hline \multirow[t]{2}{*}{ Gender } & Male & 1 & & & & & \\
\hline & Female & 0.5 & $0.19-1.25$ & 0.146 & & & \\
\hline \multirow[t]{3}{*}{ Age at onset } & EOMG & 1 & & & & & \\
\hline & LOMG & 1.2 & $0.42-3.73$ & 0.744 & & & \\
\hline & Elderly-onset MG & 0.76 & $0.18-3.90$ & 0.713 & & & \\
\hline \multirow[t]{3}{*}{ MGFA classification } & $\|$ & 1 & & & & & \\
\hline & III & 1.19 & $0.44-3.45$ & 0.741 & & & \\
\hline & IV & 0.57 & $0.09-4.58$ & 0.553 & & & \\
\hline \multirow[t]{2}{*}{ Thymoma } & Yes & 1 & & & & & \\
\hline & No & 0.71 & $0.25-1.86$ & 0.494 & & & \\
\hline \multirow[t]{2}{*}{ Thymectomy } & No & 1 & & & & & \\
\hline & Yes & 2.2 & $0.73-8.23$ & 0.192 & & & \\
\hline \multirow[t]{2}{*}{ Worsening } & Yes & & & & & & \\
\hline & No & 2.36 & $0.78-8.80$ & 0.155 & & & \\
\hline \multirow[t]{2}{*}{ Autoimmune disease } & No & 1 & & & & & \\
\hline & Yes & 0.85 & $0.22-4.21$ & 0.827 & & & \\
\hline \multirow[t]{2}{*}{ MMT score } & $>26$ & 1 & & & & & \\
\hline & $\leq 26$ & 2.36 & $0.54-9.72$ & 0.229 & & & \\
\hline \multirow[t]{2}{*}{ Anti-AChR Abs titer, nmol/L } & $>9$ & 1 & & & & & \\
\hline & $\leq 9$ & 0.49 & $0.15-1.37$ & 0.197 & & & \\
\hline \multirow[t]{2}{*}{ Duration, months } & $>12$ & 1 & & & 1 & & \\
\hline & $\leq 12$ & 4.41 & $1.73-11.90$ & $0.002^{* *}$ & 3.45 & $1.23-10.24$ & 0.021 \\
\hline \multirow[t]{2}{*}{ Pyridostigmine dosage, mg/day } & $\leq 240$ & & & & & & \\
\hline & $>240$ & 0.46 & $0.10-2.50$ & 0.339 & & & \\
\hline \multirow[t]{2}{*}{ MG-ADL score } & $>3$ & 1 & & & & & \\
\hline & $\leq 3$ & 2.74 & $0.69-18.36$ & 0.207 & & & \\
\hline \multirow[t]{2}{*}{ Bulbar score } & $\leq 1$ & 1 & & & & & \\
\hline & $>1$ & 2.01 & $0.81-5.19$ & 0.136 & & & \\
\hline \multirow[t]{2}{*}{ Respiratory function } & Normal & 1 & & & & & \\
\hline & Abnormal & 1.53 & $0.56-4.65$ & 0.426 & & & \\
\hline \multirow[t]{2}{*}{ Limb score } & $\leq 1$ & 1 & & & & & \\
\hline & $>1$ & 1.51 & $0.57-4.31$ & 0.418 & & & \\
\hline \multirow[t]{2}{*}{ Ocular score } & $>2$ & & & & 1 & & \\
\hline & $\leq 2$ & 2.87 & $1.11-8.15$ & $0.036^{* *}$ & 6.00 & $1.82-24.58$ & 0.006 \\
\hline QMG score & $\leq 13$ & 1 & & & 1 & & \\
\hline & $>13$ & 2.36 & $0.78-8.80$ & 0.155 & 11.95 & $2.31-95.82$ & 0.008 \\
\hline Extraocular muscle score & $\geq 1$ & 1 & & & & & \\
\hline & 0 & 2.52 & $0.84-9.39$ & 0.124 & & & \\
\hline Bulbar muscle score & $\geq 1$ & 1 & & & & & \\
\hline & 0 & 0.51 & $0.17-1.37$ & 0.198 & & & \\
\hline Respiratory muscle score & $\geq 80$ & 1 & & & & & \\
\hline & $65-79$ & 0.98 & $0.36-2.79$ & & & & \\
\hline & $50-64$ & 0.75 & $0.28-9.48$ & & & & \\
\hline & $<50$ & 0.81 & $0.07-16.83$ & & & & \\
\hline Gross motor score & $>9$ & 1 & & & 1 & & \\
\hline & $\leq 9$ & 2.67 & $0.90-7.81$ & $0.072^{*}$ & 10.82 & $2.22-69.13$ & 0.006 \\
\hline Axial motor score & $>2$ & 1 & & & & & \\
\hline & $\leq 2$ & 1.70 & $0.33-7.48$ & 0.493 & & & \\
\hline
\end{tabular}


Table 3 (continued)

$\mathrm{Cl}$, confidence interval, $O R$ odds ratio, MSE minimal symptom expression, EOMG early-onset myasthenia gravis, LOMG late-onset myasthenia gravis, $M G$ myasthenia gravis, MGFA Myasthenia Gravis Foundation of America, AChR acetylcholine receptor, Abs antibodies, MMT manual muscle test, MG-ADL myasthenia gravis-activity of daily living, $Q M G$ quantitative myasthenia gravis

*Statistical significance $(a=0.1)$

**Statistical significance $(a=0.05)$

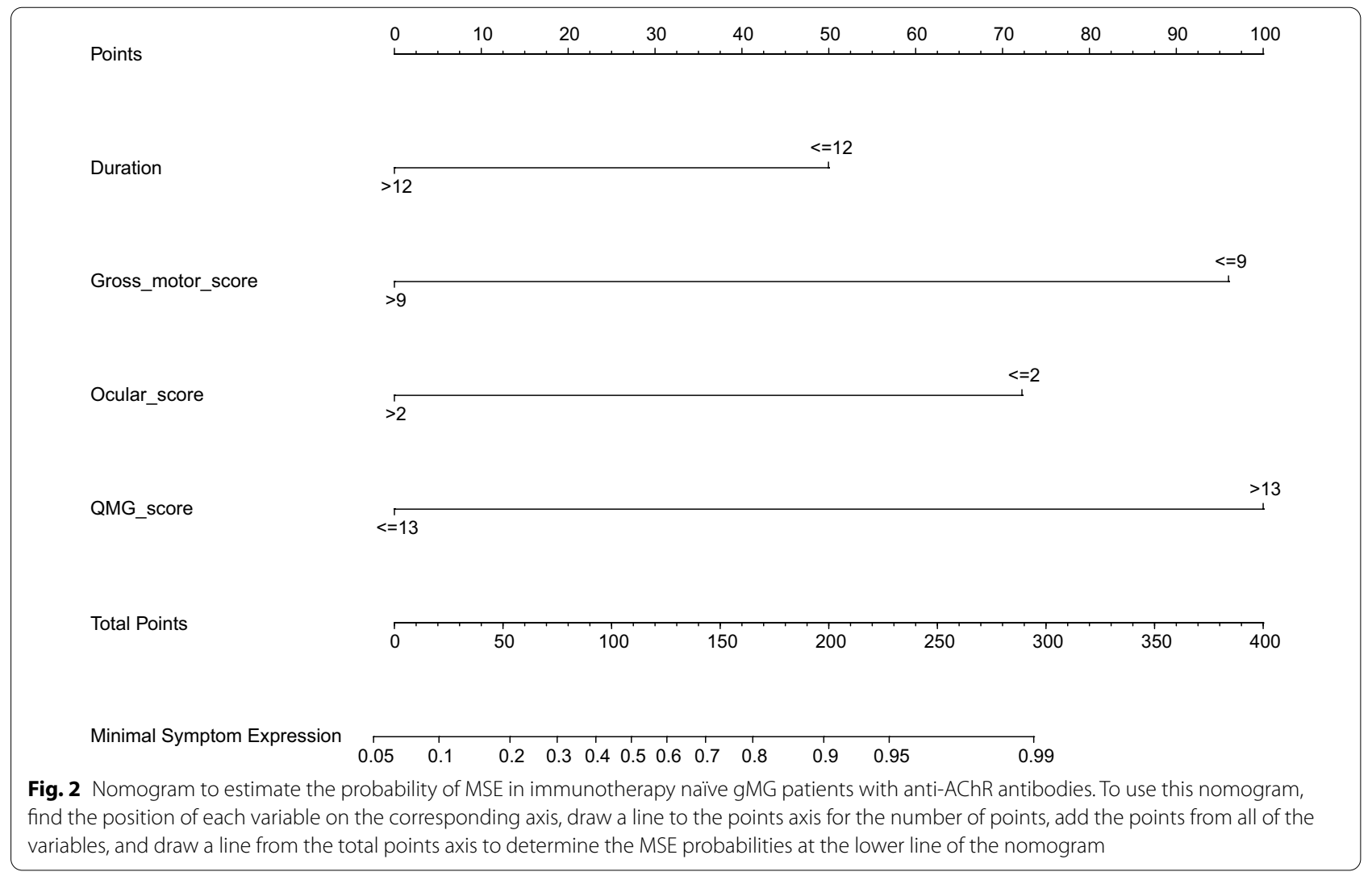
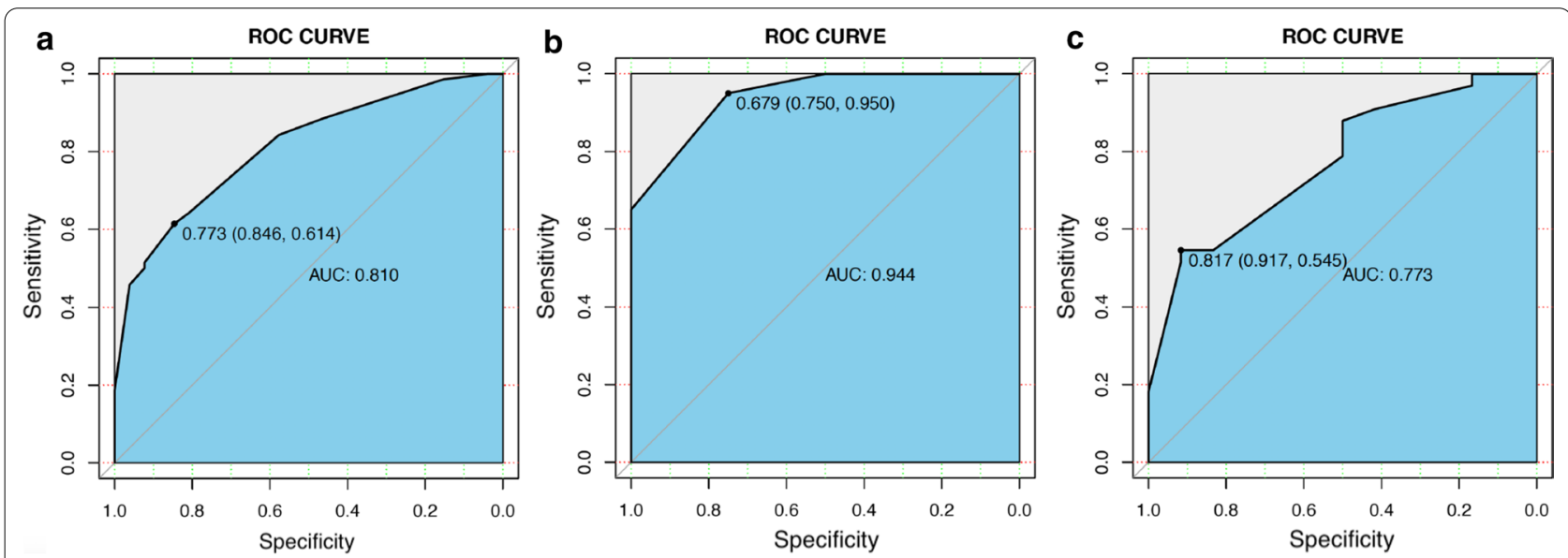

Fig. 3 The ROC curves represent the discrimination ability of the model measured by the C-index are $0.810(95 \% \mathrm{Cl}, 0.72-0.90), 0.944(95 \% \mathrm{Cl}$ 0.83-1.00), and 0.773 (95\% Cl 0.63-0.92) in the development (a), temporal validation cohort (b), and external validation cohort (c), respectively 
more frequent plasmapheresis were associated with the treatment-resistant outcome for MG patients [19]. The MIs model had good agreement with the nonimputed model and there was little difference between the ORs for the original and the MIs models. Given the high percentage of missing data, the final analyses were performed on the original model.

With the development of novel therapy for MG, MSE has severed as a patient-reported primary outcome measure in clinical trials. Tough minimal manifestation status (MMS) is the goal for the treatment of MG, MSE is more available in the clinical trials for a long followup due to its unique advantages. MSE is not only able to reflect the patient's experienced disease fluctuations symptom during a long period, but easy to acquire in an online follow-up study with no need of specialized equipment or training $[20,21]$. This study showed that the baseline characteristics before starting immunotherapy are determinants for MSE. As a result, we suggest clinical trials that use MSE as an endpoint should pay more attention to the distribution of duration, ocular score, QMG score, and gross motor score in the different groups to decrease selection bias.

There are several limitations of our study. Firstly, the records of therapy were insufficient. However, we used the statistic method including internal temporal validation and MIs to minimize these shortcomings. Secondly, patients who did not have sufficient clinical records were excluded, which may result in selection bias. Finally, the nomogram was based only on Chinese gMG patients. This nomogram may have some restrictions to predict the outcome for gMG patients from others areas due to the different treatment methods. The prospective and large-scale analysis is required to test and verify this nomogram.

\section{Conclusions}

In conclusion, we develop and validate a nomogram to predict the probability to achieve MSE using the baseline clinical characteristics. The prediction would help in clinical decision-making and prognosis monitoring. Simultaneously, we suggest that these baseline clinical characteristics should be evaluated before the selection of participants in MG trials to avoid potential bias.

\footnotetext{
Abbreviations

MG: Myasthenia gravis; NMJ: Neuromuscular junctions; gMG: Generalized myasthenia gravis; AChR: Acetylcholine receptor; MSE: Minimal symptom expression; MG-ADL: Myasthenia gravis activities of daily living; MGFA: Myasthenia Gravis Foundation of America; MMT: Manual muscle test; QMG: Quantitative myasthenia gravis; VIFs: Variance inflation factors; C-index: Concordance index; MMS: Minimal manifestation status; Cl: Confidence interval; OR: Odds ratio.
}

\section{Supplementary Information}

The online version contains supplementary material available at https://doi. org/10.1186/s12967-021-02961-9.

Additional file 1. Table S1. Univariate and multivariate Logistic regression models for minimal symptom expression in the development group.

\section{Acknowledgements}

We would like to thank Jie Song, Lei Zhou, and Jun Lu for their assistance in data collection.

\section{Authors' contributions}

RZ and YW contributed equally to this manuscript. SSL and CBZ designed the experiments; $Y W$ and $X H$ collected data; $R Z$ analyzed data; $Y W, X H, H H Z$, $J Y X, Y W D, L L, T C, Z R$, JLL, SNL, HY, YL, and SSL provided research resources; RZ wrote the manuscript; ZRZ, SSL, and CBZ reviewed and edited the manuscript. All authors read and approved the final manuscript.

\section{Funding}

This work was supported by financial grants from the National Natural Science Foundation of China (No. 81870988 and 82071410), and Shanghai Municipal Science and Technology Major Project (No.2018SHZDZX01), and ZJLab.

\section{Availability of data and materials}

The datasets used and/or analysed during the current study are available from the corresponding author on reasonable request.

\section{Declarations}

\section{Ethics approval and consent to participate}

This study was approved by the ethics committee of each hospital. Written informed consent was obtained from each study participant before sample collection. All study procedures were performed by the principles of Good Clinical Practice and the Declaration of Helsinki.

\section{Consent for publication}

Not applicable.

\section{Competing interests}

The authors declare that they have no competing interests.

\section{Author details}

${ }^{1}$ Department of Neurology, Huashan Hospital Fudan University, No.12 Middle Wulumuqi Road, Shanghai 200040, China. ${ }^{2}$ Department of Pharmacy, Huashan Hospital Fudan University, Shanghai, China. ${ }^{3}$ Radiation Oncology Center, Huashan Hospital, Fudan University, Shanghai, China. ${ }^{4}$ Department of Neurology, Xuanwu Hospital, Capital Medical University, Beijing, China. ${ }^{5}$ Department of Neurology, Tangdu Hospital, the Fourth Military Medical University, Xi'an, China. ${ }^{6}$ Department of Neurology, Wuhan No.1 Hospital, Wuhan, China. ${ }^{7}$ Department of Neurology, Xiangya Hospital, Central South University, Changsha, China.

Received: 4 May 2021 Accepted: 24 June 2021

Published online: 30 June 2021

\section{References}

1. Gilhus NE, Tzartos S, Evoli A, Palace J, Burns TM, Verschuuren J. Myasthenia gravis. Nat Rev Dis Primers. 2019;5:30.

2. Hehir MK, Silvestri NJ. Generalized myasthenia gravis: classification, clinical presentation, natural history, and epidemiology. Neurol Clin. 2018;36:253-60.

3. Lindstrom JM, Seybold ME, Lennon VA, Whittingham S, Duane DD. Antibody to acetylcholine receptor in myasthenia gravis. Prevalence, clinical correlates, and diagnostic value. Neurology. 1976;26:1054-9. 
4. Wang L, Huan X, Xi J-Y, Wu H, Zhou L, Lu J-H, Zhang T-S, Zhao C-B. Immunosuppressive and monoclonal antibody treatment for myasthenia gravis: a network meta-analysis. CNS Neurosci Ther. 2019;25:647-58.

5. Kanai T, Uzawa A, Kawaguchi N, Oda F, Ozawa Y, Himuro K, Kuwabara S. Predictive score for oral corticosteroid-induced initial worsening of seropositive generalized myasthenia gravis. J Neurol Sci. 2019;396:8-11.

6. Mao ZF, Mo XA, Qin C, Lai YR, Olde Hartman TC. Course and prognosis of myasthenia gravis: a systematic review. Eur J Neurol. 2010;17:913-21.

7. Vissing J, Jacob S, Fujita KP, O'Brien F, Howard JF."Minimal symptom expression" in patients with acetylcholine receptor antibody-positive refractory generalized myasthenia gravis treated with eculizumab. J Neurol. 2020;267:1991-2001.

8. Howard JF Jr, Bril V, Burns TM, Mantegazza R, Bilinska M, Szczudlik A, Beydoun S, Garrido F, Piehl F, Rottoli M, et al. Randomized phase 2 study of FcRn antagonist efgartigimod in generalized myasthenia gravis. Neurology. 2019;92:e2661-73.

9. Nguyen-Cao TM, Gelinas D, Griffin R, Mondou E. Myasthenia gravis: historical achievements and the "golden age" of clinical trials. J Neurol Sci. 2019;406:116428.

10. Bacci ED, Coyne KS, Poon JL, Harris L, Boscoe AN. Understanding side effects of therapy for myasthenia gravis and their impact on daily life. BMC Neurol. 2019;19:335.

11. 2020 Virtual AANEM Annual Meeting Abstract Guide. Muscle Nerve 2020, 62 Suppl 1:S1-S131.

12. Murai H, Yamashita N, Watanabe M, Nomura Y, Motomura M, Yoshikawa $\mathrm{H}$, Nakamura Y, Kawaguchi N, Onodera H, Araga S, et al. Characteristics of myasthenia gravis according to onset-age: Japanese nationwide survey. J Neurol Sci. 2011;305:97-102.

13. Hayter SM, Cook MC. Updated assessment of the prevalence, spectrum and case definition of autoimmune disease. Autoimmun Rev. 2012;11:754-65
14. Khadilkar SV, Chaudhari CR, Patil TR, Desai ND, Jagiasi KA, Bhutada AG. Once myasthenic, always myasthenic? observations on the behavior and prognosis of myasthenia gravis in a cohort of 100 patients. Neurol India. 2014;62:492-7.

15. Engel AG, Tsujihata M, Lindstrom JM, Lennon VA. The motor end plate in myasthenia gravis and in experimental autoimmune myasthenia gravis. A quantitative ultrastructural study. Ann N Y Acad Sci. 1976;274:60-79.

16. Katzberg HD, Barnett C, Bril V. Predictors of response to immunomodulation in patients with myasthenia gravis. Muscle Nerve. 2012:45:648-52.

17. Jaretzki A 3rd, Barohn RJ, Ernstoff RM, Kaminski HJ, Keesey JC, Penn AS, Sanders DB. Myasthenia gravis: recommendations for clinical research standards. Task Force of the Medical Scientific Advisory Board of the Myasthenia Gravis Foundation of America. Neurology. 2000;55:16-23.

18. Grob D, Brunner N, Namba T, Pagala M. Lifetime course of myasthenia gravis. Muscle Nerve. 2008;37:141-9.

19. Ozawa Y, Uzawa A, Yasuda M, Kojima Y, Onishi Y, Oda F, Kanai T, Himuro K, Kawaguchi N, Kuwabara S. Long-term outcomes and prognostic factors in generalized myasthenia gravis. J Neurol. 2021. https://doi.org/10.1007/ s00415-021-10520-x.

20. Muppidi S, Wolfe Gl, Conaway M, Burns TM. MG-ADL: still a relevant outcome measure. Muscle Nerve. 2011;44:727-31.

21. Muppidi S. The myasthenia gravis-specific activities of daily living profile. Ann N Y Acad Sci. 2012;1274:114-9.

\section{Publisher's Note}

Springer Nature remains neutral with regard to jurisdictional claims in published maps and institutional affiliations.
Ready to submit your research? Choose BMC and benefit from:

- fast, convenient online submission

- thorough peer review by experienced researchers in your field

- rapid publication on acceptance

- support for research data, including large and complex data types

- gold Open Access which fosters wider collaboration and increased citations

- maximum visibility for your research: over $100 \mathrm{M}$ website views per year

At BMC, research is always in progress.

Learn more biomedcentral.com/submissions 